\title{
Los límites modalmente vagos de la simplicidad mereológica
}

\section{Gonzalo Germán Núñez Erices}

Universidad Católica del Maule. Departamento de Filosofía

gnunez@ucm.cl

Fecha de recepción: $17-4-2021$

Fecha de aceptación: 1-9-2021

\section{Resumen}

Un objeto simple es un átomo mereológico, es decir, un objeto sin partes. Markosian (1998) postula lo que él llama the simple question: ¿bajo qué circunstancias es verdadero para un objeto que no posea partes? Este artículo analiza, primero, las respuestas a esa pregunta y, segundo, la tesis del nihilismo mereológico sobre composición material. Según esta última, en el mundo solo existen objetos simples que nunca componen nada. Esta tesis evita la vaguedad composicional, al no existir límites espaciales indeterminados para un objeto sin partes, pero no elimina, considerando los descubrimientos de la física cuántica, la vaguedad modal en su localización.

Palabras clave: simples; nihilismo; vaguedad; modalidad; composición material

Abstract. The modally vague boundaries of mereological simplicity

A simple object is a mereological atom, in other words an object without parts. Markosian (1998) posits what he calls The Simple Question: under what circumstances is it true of some object that it has no parts? This article analyses, first, responses to that question, and second, the nihilistic mereological thesis of material composition. According to this, there are only simple objects in the world, which never compose anything. This thesis avoids compositional vagueness, as there are no undetermined spatial boundaries of an object without parts; but taking into account the discoveries of quantum physics, it cannot get rid of the modal vagueness of its location.

Keywords: simples; boundaries; nihilism; vagueness; modality; material composition

\section{Sumario}

\section{Introducción}

2. La pregunta acerca de la simplicidad

3. Límites vagos y nihilismo mereológico

4. Indeterminación y probabilidad
5. Límites y la vaguedad modal de la simplicidad

Referencias bibliográficas 


\section{Introducción}

Varios siglos han pasado desde que pensadores como Leucipo, Demócrito o Epicuro se preguntaran por los componentes básicos e indivisibles de la materia. A pesar de que la física contemporánea ha llevado a cabo descubrimientos sorprendentes de cómo suceden las cosas a escala microscópica que desafían el sentido común, aún surgen interrogantes sobre la naturaleza de las partículas fundamentales que componen los objetos ordinarios que nos rodean. Sin embargo, el estudio sobre composición material es un tema de interés que atrae no solo a científicas(os), sino también a filósofas(os) y, en particular, a quienes se dedican a la metafísica. De acuerdo con Quine, la pregunta "¿qué es lo que hay?» exige asumir los compromisos ontológicos que estamos dispuestos a aceptar en virtud de cuantificar los componentes del mundo. Cotidianamente estamos comprometidos con la existencia de objetos materiales (como sillas, mesas, planetas o nubes) que se corresponden con la creencia de que dos o más objetos pueden componer juntos otro objeto; no solamente la aceptación de que muchos átomos componen juntos una mesa, sino también de que muchos ladrillos componen una muralla, o muchos países componen un continente. En el mundo hay muchas cosas y muchas de ellas componen juntas muchas otras más.

La ontología plantea el desafío de encontrar las justificaciones para sostener o descartar, independientemente de la cognición o el lenguaje, la existencia de este tipo de objetos materiales compuestos u otro tipo de entidad. En este aspecto, Peter van Inwagen, en su libro Material Beings (1990), plantea lo que denomina «la pregunta especial sobre la composición» (the special composition question): ¿bajo qué circunstancias muchos objetos son las partes que componen un todo? O bien, en términos de "cuantificación plural»: ¿en qué condiciones es verdadero el enunciado " $\exists$ y de modo tal que los xs componen $y$ »? Responder esta pregunta nos lleva al terreno de la mereología, es decir, al estudio de las relaciones entre las partes y el todo. En este sentido, el problema de la composición material es determinar las condiciones suficientes y necesarias para determinar si muchas cosas componen una cosa.

La pregunta especial sobre la composición presenta tres respuestas posibles que se pueden presentar de la siguiente manera: nunca, siempre y ocasionalmente. De este modo, podemos plantear este punto en términos de la siguiente pregunta: ‘hay un límite de la composición material ${ }^{1}$ ?:

i. Tesis nibilista de la composición (nihilismo mereológico). Existe un límite composicional estricto: nunca es el caso de que dos o más objetos componen otro objeto. Desde este modo, las entidades relevantes existentes son exclusivamente objetos simples (es decir, objetos sin partes) que se distribuyen espacialmente formando, en un sentido laxo, mesas, computadoras

1. En otro artículo (Núnez, 2019b) planteo con mayor precisión una lectura sobre composición material en términos estrictos como una pregunta acerca de los límites en la composición desde la cual abordo con profundidad las tres opciones aquí mencionadas. 
o árboles, pero sin implicar que tales entidades sean adicionadas a una ontología de objetos materiales.

ii. Tesis universalista de la composición (universalismo mereológico). Niega la existencia de un límite composicional: siempre es el caso de que dos o más objetos componen otro objeto. Así, no solamente existen objetos compuestos de átomos, u objetos compuestos de partes dispersas espacialmente como archipiélagos o constelaciones, sino que también existen objetos tales como aquel que se compone de tu taza de café con la luna.

iii. Tesis restrictivista de la composición (restrictivismo mereológico). Existe un límite composicional parcial: ocasionalmente es el caso de que dos o más objetos componen otro objeto. En una ontología de sentido común, existe un pueblo compuesto de muchas casas, pero no existe un objeto compuesto de tu taza de café con la luna. Desde otra perspectiva, van Inwagen (1990) y Merricks (2001) también han adoptado una posición restrictivista en la cual la composición solamente ocurre cuando la actividad de la vida está implicada. Las entidades que existen propiamente tal son simples y organismos compuestos de simples.

Cuando un límite es trazado, siempre surge la posibilidad de la vaguedad. Esta última, de hecho, puede ser explicada por la falta de claridad de un límite de modo tal que resulta indeterminado si algunas cosas están incluidas definitivamente dentro o definitivamente fuera de lo que se está delimitando. Este tipo de objetos indeterminados en relación con un límite los podemos llamar «objetos limítrofes» (borderline objects). Al trazar un límite composicional con la manifestación de actividad orgánica, el restrictivismo puede presentar casos de objetos limítrofes en los que es indeterminado si hay actividad orgánica involucrada (por ejemplo, organismos biológicos como virus, artefactos como computadoras y robots, o incluso un planeta como la Tierra que muestra procesos y ciclos internos complejos comparables a un organismo vivo). En virtud de sus posiciones extremas, tanto el nihilismo como el universalismo presentan una ventaja frente al restrictivismo: no hay espacio para la vaguedad. Por un lado, según el universalismo, no existen objetos limítrofes porque, siempre que dos o más objetos existen, un objeto compuesto por ellos existe también. Por otro lado, según el nihilismo, no existen objetos limítrofes porque solo existen objetos simples que nunca componen nada entre sí.

Este artículo estudia particularmente los objetos simples como las entidades primordiales que considera el nihilismo. Hay tres instancias fundamentales. Primero, se explora la pregunta acerca de la simplicidad (the simple question) planteada por Ned Markosian en su artículo «Simples» (1998). Las respuestas abordadas en dicho artículo acerca de las condiciones necesarias y suficientes para que una entidad sea considerada un objeto simple son abordadas desde dos tesis diferentes: la tesis de la indivisibilidad y la tesis de la espacialidad. Segundo, se plantea el tema de la vaguedad ontológica en términos de objetos con límites vagos y cómo el nihilismo evita la existencia de ese tipo de objetos. Finalmente, con la incorporación de algunas consideraciones desde la física 
cuántica, se defiende la siguiente tesis: aunque el nihilismo evita la introducción de la vaguedad en términos composicionales, no evita la vaguedad en términos modales. Un objeto simple es vago en la medida en que posee límites modales en lo que resulta indeterminado, entre todos los mundos posibles, cuál de ellos precisa la posición actual de un objeto simple.

\section{La pregunta acerca de la simplicidad}

Si el nihilismo es la respuesta correcta a la pregunta especial sobre la composición material, los simples son entonces las piezas ontológicas exclusivas que constituyen el mundo. En términos mereológicos, un simple presenta la siguiente definición:

$x$ es una parte (genuina) de $y={ }_{\mathrm{df}} x$ es una parte de $y$, e $y$ es distinto a $x$. $x$ es un simple $=_{\mathrm{df}} x$ es un objeto que carece de partes genuinas ${ }^{2}$.

Aunque un objeto simple puede ser parte de un objeto compuesto, nada puede ser parte de un simple. Sin embargo, ¿cuáles son las condiciones necesarias y suficientes para la simplicidad de un objeto? Esta pregunta puede ser abordada desde dos perspectivas de análisis: la tesis de la indivisibilidad y la tesis de la espacialidad de la simplicidad.

\subsection{La tesis de la indivisibilidad}

La simplicidad es un caso de indivisibilidad que se deriva naturalmente del

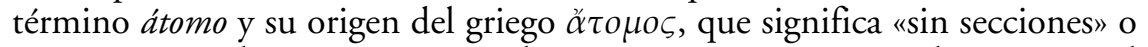
"que no se puede cortar». Un simple, por consiguiente, es un objeto material extenso sin partes físicas. Esto se puede colocar en los siguientes términos:

(TIF) Tesis de indivisibilidad fisica de la simplicidad: necesariamente, $\mathrm{x}$ es un simple si y solo si resulta imposible dividir físicamente $\mathrm{x}$ en partes.

Según la TIF, los simples son entidades físicas que ocupan una porción limitada de espacio. Ellos son los bloques fundamentales de la construcción del mundo: allí donde la división de la realidad se detiene y la materia no puede ser cortada en piezas más pequeñas. La cadena de divisibilidad de los objetos materiales no puede ser llevada ad infinitum si los simples existen como entidades que no pueden ser físicamente divididas en otras partes. Podemos concebirlos como objetos con límites indestructibles o, por lo menos, cuya destrucción implicaría la destrucción del objeto en cuanto tal.

No obstante, el hecho de que una entidad satisfaga las características de la TIF no implica necesariamente que una entidad sea un objeto simple. Tal como es argumentado por Markosian (1998), podemos imaginar una cadena

2. Considerando como una verdad trivial el hecho de que cada objeto se compone a sí mismo, es decir, cada objeto es una parte de sí mismo. 
hecha de algún material desconocido que resulte físicamente imposible de fragmentar en pedazos más pequeños, de modo tal que, de acuerdo con la TIF, este tipo de objeto sería un simple. Sin embargo, el problema es el siguiente: aunque la cadena es indivisible, es claro que está compuesta de partes que son sus eslabones y, por ende, no es un objeto simple (por lo menos no en un sentido mereológico). Podemos concebir objetos materiales compuestos a pesar de que sus superficies sean límites físicamente impenetrables. Ante esta situación, la simplicidad no sería un asunto de indivisibilidad física, sino de indivisibilidad metafísica:

(TIM) Tesis de indivisibilidad metafisica de la simplicidad: necesariamente, $\mathrm{x}$ es un simple si y solo si x no puede ser metafísicamente dividido o descompuesto en partes.

Esta definición requiere clarificar lo que significa «división metafísica». Para ensayar una explicación posible podemos acudir al Tractatus de Wittgenstein (1921): «El objeto es simple» (2.02); «Los objetos forman la substancia del mundo. Por eso no pueden ser compuestos» (2.021). En este caso, los simples no son entendidos como un tipo de entidad físicamente indivisible, sino como un tipo de entidad abstracta que cumple un rol fundacional en la arquitectura metafísica del mundo. Los simples son el resultado del análisis lógico capaz de descomponer el mundo y el lenguaje desde sus estructuras más complejas hasta sus elementos más simples. Ambos comparten lo que Wittgenstein denomina una "forma lógica de figuración» (cf., 1921: 2.2), de modo tal que las proposiciones son un modelo o figura de los hechos. Mientras el mundo es la totalidad de los hechos que se pueden descomponer en estados de cosas o hechos atómicos cuyos componentes son objetos simples, el lenguaje es la totalidad de proposiciones complejas que pueden ser completamente analizadas hasta proposiciones elementales cuyos componentes últimos son nombres o signos primitivos.

Los simples, por consiguiente, son átomos metafísicos como resultado de un postulado a priori en virtud del análisis y la descomposición lógica del lenguaje y el mundo. Como sostiene Wittgenstein, «si el mundo no tuviera sustancia alguna, el que una proposición tuviera sentido dependería de que otra proposición fuera verdadera" (1921: 2.0211). Dicho de otro modo, de acuerdo con el autor vienés, la existencia de objetos simples fijados en el fondo de la realidad garantiza, a su vez, el hecho de que el análisis lógico de una proposición no dependa infinitamente de otra proposición menos compleja. Así, puesto que existen objetos simples, entonces existen también proposiciones elementales o inanalizables cuyo sentido se desprende de su propia naturaleza lógica dada la existencia del hecho atómico particular que le entrega su valor de verdad (es decir, la posibilidad lógica de que tales proposiciones puedan ser verdaderas o falsas). En definitiva, la existencia de objetos simples o substancias atómicas es, para Wittgenstein, la necesidad metafísica de evitar una progresión infinita en el análisis lógico del lenguaje y la descomposición 
del mundo. En este subnivel atómico, el análisis y la descomposición alcanzan un límite metafísicamente insuperable. En este sentido, la TIM puede ser replanteada del siguiente modo:

Necesariamente, $\mathrm{x}$ es un simple si y solo si $\mathrm{x}$ es un elemento analíticamente indivisible del mundo de modo tal que no puede ser lógicamente descompuesto en otros elementos.

Los simples, desde esta perspectiva atomista, son entidades metafísicas particulares o únicas que se caracterizan, como ninguna otra, por ser ontológicamente independientes: su existencia no está justificada en la existencia de otro tipo de entidad, sino en la profunda y última necesidad de cómo es el mundo. En este sentido, Russell sostiene «la justificación del atomismo lógico, la perspectiva que puedes alcanzar en teoría, si no es en la práctica, de objetos simples últimos con los cuales el mundo es construido, y esos simples poseen un tipo de realidad que no es propia a nada más» (1918: 142). Sin embargo, en términos epistemológicos, hay que destacar que el pluralismo ontológico russelliano, en contraposición al holismo lógico neohegeliano, es escéptico respecto del conocimiento que podemos tener de entidades metafísicamente simples o átomos lógicos. Desde el punto de vista del lenguaje, la clásica teoría de la referencia que sostiene Russell en "On Denoting» (1905) se basa en una distinción entre conocimiento por descripción (by description) y por referencia (by acquaintance). Por una parte, los nombres propios ordinarios o gramaticales (por ejemplo, Aristóteles o mesa) se refieren indirectamente a un objeto en virtud de todas las descripciones posibles asociadas. Por otro lado, un nombre propio lógico (demostrativos o indexicales tales como eso, esto, aquello, etcétera) se refiere directamente a un objeto sin descripciones mediante. En este sentido, el uso de un nombre propio lógico no garantiza para Russell la posibilidad de un conocimiento metafísico de algún tipo de objeto simple como referencia. Más bien todo nombre propio lógico denota ya sea universales (por ejemplo, relaciones, propiedades o proposiciones) o bien datos de los sentidos (sense data) que son contenidos mentales. En definitiva, previamente a su adopción a un monismo neutro como una mejor respuesta filosófica al problema mente-cerebro, Russell ya mostraba una versión escéptica acerca de las posibilidades del conocimiento humano para capturar metafísicamente la existencia de objetos simples con independencia de la realidad mental.

Finalmente, tal como hemos caracterizado la TIM, los simples no son objetos sin partes en el espacio físico de acuerdo con la TIF, sino objetos atómicos ontológicamente indivisibles en el espacio lógico. Sin embargo, entre muchas de las razones por las cuales el atomismo lógico es una teoría metafísica abandonada en el tiempo, están los propios descubrimientos de la física cuántica. Estos han demostrado, entre otras cosas, que no existen entidades ontológicamente independientes cuya existencia sea absolutamente causa sui. El fenómeno físico de "entrelazamiento cuántico» (quantum entaglement), por ejemplo, ocurre cuando un grupo de partículas subatómicas se encuentran en 
una proximidad espacial de tal modo que las propiedades del estado cuántico de una de ellas no pueden ser descritas de manera independiente de las propiedades del resto de las otras partículas. El entrelazamiento es un sistema cuántico de carácter holista en el cual no existen partículas individuales, sino un todo de correlaciones causales y también estocásticas. En este sentido, la información medible de una partícula (por ejemplo, su unidad de momentum) no puede ser completada sin la información del resto de los constituyentes básicos del sistema. Por lo tanto, cuánticamente, a diferencia de las exigencias metafísicas de independencia ontológica de los objetos simples postulados por el atomismo lógico, existe un principio de symploké o interconexión radical entre las cosas, ya descrito por Platón en El sofista, según el cual resulta insostenible la idea de la existencia de objetos metafísicamente simples que dependan, en algún tipo de dimensión ontológica, exclusivamente de sí mismos ${ }^{3}$. La consistencia filosófica de la teoría de la simplicidad que podemos obtener en la TIM no puede, en este aspecto, descansar únicamente en el análisis lógico, sino también, como nos plantea Quine, en los compromisos ontológicos adoptados a partir de los descubrimientos que la física cuántica nos pueda entregar.

\subsection{La tesis de la espacialidad}

La simplicidad para un objeto implica características espaciales en lugar de condiciones sobre su indivisibilidad. Una opción es establecer que los simples no poseen extensión, ya que fallarían en existir en algunas de las dimensiones espaciales. Esta es la tesis geométrica de la simplicidad:

(TG) La tesis geométrica de la simplicidad: $\mathrm{x}$ es un simple si $\mathrm{y}$ solo si $\mathrm{x}$ es un objeto-punto ${ }^{4}$.

Que un objeto sea simple significa que la región de espacio que ocupa está completamente saturada por un punto. Sin embargo, si los objetos físicos son objetos que necesariamente existen extendidos en el espacio, una opción como la TG resultaría insatisfactoria, ya que un punto no tiene dimensionalidad espacial al no poseer largo, ancho ni altura. Existir en alguna de estas dimensiones espaciales conlleva para un objeto poseer una estructura mereológica (es

3. A pesar de este problema planteado en la teoría de la simplicidad que podemos encontrar en el atomismo lógico, el hecho de que Wittgenstein en el Tractatus da cuenta de lo que podríamos llamar un sistema de entrelazamiento en proposiciones como las siguientes: "Poder ser parte integrante de un estado de cosas es esencial a la cosa»; «En la lógica nada es causa: si la cosa puede ocurrir en el estado de cosas, la posibilidad del estado de cosas tiene que venir ya prejuzgada en la cosa» (1921: 2.011-2.012). Un objeto simple tractariano, de acuerdo con esta descripción, solo puede existir en la medida en que es parte de un sistema de relaciones lógicas con otros objetos. Esto es, la existencia de un objeto depende de las correlaciones de las combinaciones posibles con otros objetos en la ocurrencia de estados de cosas.

4. Markosian (1998) denomina esta tesis como the pointy view of simples. 
decir, una relación todo-partes). Para cada objeto que se extiende en alguna de las dimensiones espaciales, este puede ser dividido en partes que se extienden en la misma dimensión espacial en la cual tal objeto existe, pero con un límite de una dimensión espacial menor. Así, objetos tridimensionales poseen partes de tres dimensiones y superficies como límites; objetos bidimensionales poseen partes de dos dimensiones y líneas como límites; y objetos unidimensionales poseen partes de una dimensión y puntos como límites. Si un objeto extenso es una entidad que posee límites a lo largo de alguna de las dimensiones espaciales, un punto es una entidad que no posee un límite en cuanto que no existen límites con una dimensionalidad espacial menor. Si estos no tienen límites, entonces los puntos son objetos sin extensión espacial que no pueden ser divididos en partes cero dimensionales. De esta manera, la geometría euclidiana es construida sobre estos elementos primitivos que no pueden ser definidos en virtud de otros objetos. Si la TG es correcta, entonces los simples son objetos sin dimensionalidad espacial y, por ende, sin límites (o, por lo menos, límites en virtud de alguna dimensión espacial).

Dado el razonamiento anterior, la TG mostraría un problema similar a la TIM: en ambas tesis los simples resultan ser entidades no físicas en cuanto que existen en un plano metafísico o en un plano abstracto geométrico. Por consiguiente, tendríamos razones para descartar la existencia física y espacial de simples. A pesar de esto, Markosian sostiene lo siguiente: «Parece perfectamente posible que pudiéramos tener evidencia que hay objetos punto, en la forma de la mejor teoría física que postula objetos sin extensión, pero con características que los haría parecer clasificados apropiadamente como físicos» (1998: 217). En este sentido, los simples son objetos-puntos como resultado de una idealización o proceso de abstracción de las partículas fundamentales estudiadas por la física cuántica, las cuales, a pesar de no tener una extensión espacial u ocupar una región exclusiva en el espacio a un tiempo determinado, no dejan de ser algo material con poderes causales físicos.

La principal objeción a la TG, planteada en este caso por Markosian, es que, si esta fuese verdadera, entonces no es posible un mundo en el cual solamente exista un objeto físico. Imagina en tal mundo la existencia de un objeto material $\mathrm{O}$ que es una esfera perfectamente sólida. Así, $\mathrm{O}$ es un objeto espacialmente continuo hecho de una sustancia material homogénea; $\mathrm{O}$ llena una región de espacio $\mathrm{R}$ donde no hay subregiones de $\mathrm{R}$ vacías y desconectadas entre sí, es decir, en $\mathrm{R}$ hay materia por todos lados sin dejar ningún rincón sin llenar. En este caso, si $\mathrm{R}$ es una porción de espacio llenada continuamente o saturada con materia, entonces la aceptación de la TG implicaría que en $\mathrm{R}$ existen infinitos objetos: o bien (i) hay infinitos objetos simples en $\mathrm{R}$ que componen $\mathrm{O}$, o bien (ii) hay infinitos objetos no simples en $\mathrm{R}$ que componen $\mathrm{O}$.

En cuanto a (i), O estaría compuesto de lo que en la literatura se denomina atomless gunk, es decir, cada parte que compone a $\mathrm{O}$ está compuesta, a la vez, de otras partes. Por ende, si cada parte que compone a $\mathrm{O}$ tiene una parte, entonces en R existen infinitos objetos. En cuanto a (ii), si la TG es verdadera, entonces una cantidad finita de puntos para componer $\mathrm{O}$ no sería suficiente 
para que $\mathrm{R}$ sea una región espacial llenada o saturada continuamente con materia. Por lo tanto, O debe componerse de infinitos objetos simples, es decir, objetos-punto, de modo tal que no queden espacios sin llenar en R. En conclusión, si la TG es verdadera, entonces, existirían dos proposiciones incompatibles entre sí: primero, «un objeto material con una extensión espacial continua está compuesto por un número infinito de objetos»; segundo, «no es posible la existencia en el mundo de un solo objeto físico espacialmente extenso». Dado que es posible imaginar un mundo donde existe un único objeto extenso, la TG no es aceptable: allí donde pensamos que hay un solo objeto, en realidad existen infinitos objetos. Esto lleva a Markosian a postular otra respuesta a la pregunta sobre la simplicidad:

(MaxCon) La tesis de la continuidad maximalista de la simplicidad: necesariamente, $\mathrm{x}$ es un simple si $\mathrm{y}$ solo si $\mathrm{x}$ es un objeto maximalistamente continuo. (cf., 1998: 222)

Un objeto físico $\mathrm{O}$ que llena materialmente una región espacial $\mathrm{R}$ es maximalistamente continuo en las siguientes condiciones: (i) $\mathrm{R}$ no es un subconjunto genuino de alguna región espacial más extensa que R; (ii) $\mathrm{R}$ está enteramente llenada o saturada de la materia que compone $\mathrm{x}$ (es decir, $\mathrm{R}$ no tiene subregiones sin materia). En definitiva, de acuerdo con MaxCon, un simple es un objeto físicamente extendido que llena con materia continuamente todos los rincones de la región de espacio que ocupa.

MaxCon no es una respuesta intuitiva a la pregunta sobre la simplicidad. A diferencia de la TG, en la que los simples son objetos-punto sin ningún tipo de dimensión espacial, un simple, de acuerdo con MaxCon, es un objeto espacialmente extenso que no es definido en términos de tamaño o forma, sino exclusivamente en términos de su ocupación maximalista de una región espacial. Si el requisito principal para que un objeto sea simple es saturar continuamente con materia una región espacial sin ser parte de otra región espacial más extensa, entonces un simple puede tener cualquier tamaño o forma. MaxCon permite que un simple o átomo mereológico pueda ser tan pequeño como una partícula física o tan grande como un planeta.

La TG puede plantear una objeción a MaxCon: si un objeto es espacialmente extendido, entonces tiene por lo menos dos mitades; por lo tanto, ese objeto no puede ser simple ya que un simple no tiene partes. Markosian (1998: 223) rechaza la primera premisa del argumento puesto que rechaza lo que van Inwagen (2001) denomina the doctrine of arbitrary undetached parts (DAUP) que, en su terminología básica, sostiene lo siguiente:

DAUP: para cada objeto material $\mathrm{O}$, un tiempo dado $\mathrm{t}$, $\mathrm{y}$ regiones espaciales $\mathrm{R}$ y $\mathrm{R}^{*}$, si $\mathrm{O}$ ocupa $\mathrm{R}$ en t y $\mathrm{R}^{*}$ es una subregión de $\mathrm{R}$ en $\mathrm{t}$, entonces existe una parte de $\mathrm{O}$ que ocupa $\mathrm{R}^{*}$ en $t$.

Dada su posición restrictivista brevemente mencionada en la introducción, van Inwagen rechaza la DAUP en virtud de la arbitrariedad de asignar una 
estructura mereológica a un objeto material inanimado que ocupa una determinada región espacial. Solamente objetos que incluyen una actividad vital pueden estar genuinamente compuestos de partes sin incurrir en arbitrariedades. Por lo tanto, si existen razones justificadas para rechazar la DAUP, entonces pueden existir simples según MaxCon sin importar el tamaño o la forma de la región espacial que ocupan.

En términos generales, se han explicado diferentes respuestas a la pregunta sobre la simplicidad ${ }^{5}$. A pesar de sus diferencias, sí parecen acordar que la simplicidad es un problema, entre otras cosas, acerca de límites. Por un lado, como sabemos, tanto la TIF como la TIM definen la simplicidad en términos de indivisibilidad. Según la primera, un objeto simple posee un límite físicamente impenetrable que no permite separarlo en partes; según la segunda, un objeto simple es metafísicamente indivisible cuando existe un límite lógico para la descomposición analítica del mundo. Por otro lado, la TG y MaxCon definen la simplicidad en términos espaciales. Con la primera, los simples no poseen extensión espacial, puesto que objetos-punto, al no tener límites en ninguna de las dimensiones espaciales, no pueden ser divididos en partes. La simplicidad implica la postulación de objetos sin límites. Finalmente, MaxCon acepta la existencia de objetos simples extensos de cualquier tamaño y forma, puesto que ocupan maximalistamente el espacio. Esto es, encontrar un objeto simple implica encontrar un límite rígido y preciso para una región espacial tridimensional continua que contenga y comprima toda la materia que compone dicho objeto sin dejar espacio vacío alguno. En este sentido, ya sea por su indivisibilidad o características espaciales, un simple es un objeto cuyos límites se caracterizan por la indestructibilidad, rigidez y precisión. Por consiguiente, para un simple, cualquiera que sea la teoría filosófica que explica su naturaleza, no podría haber vaguedad en sus límites.

\section{Límites vagos y nihilismo mereológico}

Entre los objetos materiales que podemos contabilizar en el mundo cotidiano a escala humana, algunos de ellos, podemos decir, poseen límites vagos. Por ejemplo, desiertos, nubes, constelaciones, bandadas, cardúmenes, el humo de un cigarro, la copa de un frondoso árbol o montañas son, entre otros, objetos vagos en cuanto no hay un límite claro y preciso entre ellos y sus ambientes o entornos espaciales. En particular, hay un lugar claro en el cual estamos en un desierto y un lugar claro en el cual dejamos de estar. No obstante, no hay un límite claro que podamos identificar dónde termina un lugar y donde comienza el otro lugar. A diferencia de nuestra percepción de una bola de billar con su superficie perfectamente compacta, pulida y lisa, en el borde de un desierto pode-

5. Existe, sin embargo, otra respuesta a la pregunta por la simplicidad no considerada en este artículo, pero tan importante como las que han sido ya revisadas. Esta sostiene que la simplicidad es bruta (brute) o, en cierto modo, primitiva. Esta es la perspectiva defendida por McDaniel: «No hay un criterio correcto, finitamente estable, o no circular para ser un simple» (2007: 233). 
mos encontrar porciones de arena limítrofes que no son definitivamente partes del desierto ni definitivamente no partes de los alrededores del desierto. Por consiguiente, un objeto vago se puede definir como un objeto de límites indefinidos, de modo que, para todo objeto físico $\mathrm{O}$, una colección de objetos $\mathrm{xs}$, una región espacial $\mathrm{R}$, un tiempo t y un límite vago $\beta$, entonces:

$\mathrm{O}$ es un objeto de límites borrosos si y solo si (i) $\mathrm{O}$ ocupa materialmente $\mathrm{R}$ y cada parte de $\mathrm{O}$ ocupa una subregión de $\mathrm{R}$ en $\mathrm{t}$, (ii) $\beta$ es una zona de transición que separa $\mathrm{R}$ del entorno espacial de R, y (iii) cada uno de los xs se encuentra en $\beta$ de modo tal que es indeterminado si son miembros de $\mathrm{R} o$ miembros del entorno espacial de $\mathrm{R}$ en $\mathrm{t}$.

La vaguedad puede ser explicada desde tres dimensiones diferentes. En primer lugar, la tesis lingüística sostiene que un predicado $F$ es vago porque hay un límite borroso en el cual es indeterminado si un objeto $a$ es $F$ o es no-F. Así, el término desierto no es capaz de precisar la aplicación clara respecto de los objetos que caen dentro de su dominio. En segundo lugar, la tesis epistémica sostiene que la vaguedad no es el resultado de las ambigüedades conceptuales, sino de nuestra ignorancia. Esto es, un predicado vago tiene, de hecho, un límite preciso que define la extensión de su valor semántico (alto se distingue con precisión de bajo), solo que desconocemos dónde está trazado dicho límite. Finalmente, la tesis ontológica u óntica sostiene que la vaguedad no es una cuestión de límites semánticos imprecisos o el desconocimiento de un límite preciso, sino simple y llanamente una existencia bona fide de objetos con límites vagos ${ }^{6}$.

Aunque la vaguedad óntica podría aplicar de manera más evidente a objetos como nubes o desiertos, desde un punto de vista microscópico, las superficies de todos los objetos físicos ordinarios -incluso aquellos que aparentan poseer un superficie suave y lisa (por ejemplo, una bola de billar, una pieza de mármol pulido, etcétera) — son en realidad enjambres atómicos irregulares y, como plantea Simons, «en el mejor de los casos, tenemos las superficies de los átomos democritianos, pero estos están espacialmente separados sin formar un todo conectado" (1991: 91). Desde esta perspectiva, todo objeto físico ordinario es un objeto vago ${ }^{7}$. Si no hay una restricción clara y precisa en la composición material, el mundo sería un lugar vago poblado por objetos limítrofes. Esto incluso si aceptamos el restrictivismo adoptado por van Inwagen y Merricks en la medida en que «una vida limítrofe [borderline] es, por definición, un evento del cual no es definitivamente verdadero y definitivamente falso que es una vida» (van Inwagen, 1990: 278). En este sentido, posiciones eliminativistas y radicales respecto de la composición material, tales como el

6. Filósofos tradicionalmente han rechazado la tesis ontológica de la vaguedad en favor de las otras dos tesis mencionadas Ver, por ejemplo, Lewis (1986: 212), Heller (2008: 51), Dummett (1999: 111), Russell (1923: 62).

7. Referencia a un artículo propio que estudia la vaguedad de los límites bona fide de los objetos materiales ordinarios que, por razones de esta versión anonimizada, es por ahora suprimida. 
nihilismo mereológico, podrían escapar a la vaguedad ontológica que implica hacer flexible, en cualquier grado, el límite que se establece cuando dos o más cosas componen algo más.

La tesis sobre una vaguedad ontológica ha recibido algunas críticas importantes sin necesariamente endorsar compromisos eliminativistas con objetos materiales ordinarios. Entre las más clásicas, se encuentra la planteada por Gareth Evans en un breve pero agudo artículo titulado "Can There Be Vague Objects?» (1978). Inspirado en el famoso ataque de Kripke a la identidad contingente en lo que respecta al problema mente-cerebro, Evans sostiene que la vaguedad $\mathrm{o}$ indeterminación óntica que involucra identidad del tipo $\mathrm{a}=\mathrm{b}$ es lógicamente incoherente. En términos generales, el argumento plantea las siguientes premisas:
(i) $\quad \nabla \mathrm{a}=\mathrm{b}$
(ii) $\quad x[\nabla(x=a)] b$
(iii) $\neg \nabla(\mathrm{a}=\mathrm{a})$
(iv) $\neg \mathrm{x}[\nabla(\mathrm{x}=\mathrm{a})] \mathrm{a}$
(v) $\quad \neg(\mathrm{a}=\mathrm{b})$

En el caso de (i), el operador $\nabla$ es utilizado para afirmar la proposición según la cual es indeterminado que a y b sean idénticas entre sí. Ahora bien, puesto que la identidad es una relación que se establece entre las propiedades de las cosas, en el caso de (ii) se establece que b tiene la propiedad de ser indeterminadamente idéntica con a, mientras que (iii) expresa la negación de la indeterminación de a de ser idéntica con a en la medida en que toda entidad tiene, necesariamente, la propiedad de ser idéntica a sí misma. Sin embargo, dado que la premisa (iv) afirma que no es el caso que a tiene la propiedad de ser indeterminadamente idéntica con a, entonces (v) concluye que, dada la ley de Leibniz, según la cual dos entidades son idénticas si tienen las mismas propiedades, no es cierto que a y b tienen una relación de identidad, puesto que fallarían en poseer las mismas propiedades: mientras que a tiene la propiedad de ser necesariamente idéntica con a, b tendría la propiedad de ser indeterminadamente idéntica con b. Por consiguiente, la identidad de a y $\mathrm{b}$ no es indeterminada, sino definitivamente falsa. El argumento de Evans desestima la vaguedad ontológica, ya que las premisas que sostienen su justificación son lógicamente inconsistentes entre sí, tal como se evidencia en (i) y (ii).

Si bien el argumento de Evans es lógicamente atractivo, no es interés de este artículo analizar las justificaciones lógicas para rechazar la vaguedad ontológica, sino las razones modales con las cuales podemos desestimar la ausencia de vaguedad en las entidades simples que defiende el nihilismo mereológico como únicos componentes bona fide de la realidad material. La argumentación de Evans, en su razonamiento lógico impecable, lleva la discusión sobre vaguedad ontológica a una reductio ad absurdum ya que apela a la verdad analítica y, por consiguiente, tautológica de que, necesariamente, toda entidad es idéntica a sí misma. No obstante, algunas posiciones filosóficas han planteado que 
la lógica debería ser ontológicamente neutral ( $c f$., Cowles y White, 1991), es decir, las verdades que son lógicamente demostrables no deberían tener una implicancia inmediata (o, incluso, necesaria) en las verdades que podamos establecer acerca de algún tipo de ontología.

En este sentido, la justificación racional de una tesis sobre vaguedad ontológica que considera descubrimientos de mecánica cuántica desafía también los principios lógicos que sostienen el argumento de Evans. En cuanto a objetos macroscópicos materiales, la ley de Leibniz, a la que apela Evans, parece ser un principio lógico seguro y razonable: si dos objetos instancian exactamente las mismas propiedades, entonces son idénticos entre sí. De este modo, aplicando la navaja de Ockham para evitar multiplicar innecesariamente las entidades en el mundo, si los términos a y b son idénticos entre sí, entonces, necesariamente, refieren a una sola y misma entidad. Sin embargo, cuánticamente, podemos observar dos partículas (fermiones o bosones) que, aunque comparten exactamente las mismas propiedades intrínsecas (por ejemplo, carga eléctrica, spin, masa, etcétera) y también sus propiedades relacionales, de modo tal que ninguna medición posible podría determinar cuál es cuál, no nos permiten aplicar la ley de Leibniz para reducir lógicamente la cantidad de objetos en el mundo ${ }^{8}$. Así, la identidad de los indiscernibles puede ser postulada, más bien, como contingentemente verdadera en ciertos casos donde aplica las leyes del pensamiento lógico, pero no puede ser extendida directamente a toda dimensión ontológica donde aplica, por ejemplo, las leyes de la física cuántica. Por lo demás, podemos considerar aspectos de indeterminación cuántica, tal como veremos más adelante, como formas de vaguedad en la naturaleza que no dejan existir por el hecho de que la lógica pueda refutarlo con sus tautologías.

Regresando al tema de interés de este artículo sobre composición material, la tesis nihilista puede ser pensada, de hecho, como una posibilidad teórica de rechazo a la vaguedad ontológica. Consideremos el argumento planteado por Peter Unger (1980) respecto de lo que él denomina The Problem of Many. Este se basa en una reformulación de las paradojas sorites: si una piedra ocupa una región de espacio $\mathrm{R}$ con una cantidad finita de átomos y removemos un átomo $\mathrm{R}$, entonces $\mathrm{R}$ estará ocupada por una piedra con N-1 átomos; si continuamente removemos uno a uno los átomos de $\mathrm{R}$ y ninguna remoción destruye la piedra, entonces llegará un momento en el que existirá una piedra en $\mathrm{R}$ sin ningún átomo, cuestión que, por supuesto, resulta ser absurda. Sin embargo, en las cercanías del borde de R (stopping place, en palabras de Unger), existen muchos átomos limítrofes que no son definitivamente ni parte de $\mathrm{R}$ ni noparte de R. Puesto que hay un imposibilidad ontológica de precisar un límite para $\mathrm{R}$ y sería ilógico pensar que existe una piedra en un región de espacio donde no hay átomos, entonces resulta conveniente desestimar la existencia de objetos materiales compuestos de cualquier tipo (por ejemplo, piedras,

8. Revisar Lowe (1999) para más detalles sobre vaguedad en la identidad y su contraste con indeterminación cuántica. 
sillas, vasos, personas, etcétera) y solo sostener la existencia de objetos simples (átomos mereológicos), es decir, objetos sin partes.

La tesis nihilista es radical y contraintuitiva en la medida en que solo reconoce a los simples como los objetos genuinos que constituyen el mundo. Estos, en rigor, aunque sí son capaces de distribuirse espacialmente en forma de piedras o mesas, aquello no implica la existencia de una piedra o una mesa compuesta por ellos. El nihilismo evita, de esta manera, cualquier vaguedad ontológica. En una ontología de sentido común, una colección de objetos compone un objeto (por ejemplo, una mesa está compuesta de todas sus partes). Sin embargo, en otros casos, no está definido claramente si dos o más objetos componen un solo objeto. Por ejemplo, si doscientos mil árboles definitivamente componen un bosque, mientras que cinco árboles definitivamente no lo hacen, entonces, ¿cuál es el número mínimo de árboles para que exista un bosque? Tanto el nihilista como el universalista no necesitan enfrentar ese tipo de paradojas sorites: para el primero, la composición nunca ocurre y, por lo tanto, no existen ni bosques ni árboles, solo objetos simples; para el segundo, no hay límite en la composición y, por ende, bastan dos árboles para que exista una tercera entidad compuesta por aquellos. Así, ambas respuestas radicales evitan la vaguedad ontológica en la medida en que la composición o bien ocurre nunca o bien ocurre siempre, lo cual no deja espacio para objetos limítrofes?

Considerando nuevamente al argumento de the problem of many, la vaguedad surge tanto de la composición como de la localización. En primer lugar, existen tantos átomos limítrofes esparcidos en los alrededores espaciales de una piedra perfectamente pulida que resulta indeterminado si algunos de ellos son parte o no de la superficie de la piedra. Si consideramos algunos de ellos, obtenemos una superficie y, si consideramos otros, obtenemos otra superficie. ¿Qué superficie es entonces el límite genuino de la piedra? Por un lado, podemos escoger una sola superficie compuesta por ciertos átomos, cuestión que parece ser arbitraria: ¿por qué esa superficie compuesta de esos átomos y no otra superficie compuesta de otros átomos ligeramente diferentes? Por otro lado, podemos escoger millones de superficies como resultado de todas las combi-

9. El nihilismo mereológico tiene que enfrentar no solamente problemas sobre vaguedad que estoy tratando en este artículo, sino también posiciones emergentistas que son relevantes de mencionar. Puede ser el caso que una colección de objetos que se encuentran distribuidos espacialmente de un modo tal que juntos no solamente componen un objeto adicional, sino que también algún tipo de propiedades emergentes son instanciadas por este último. Estas propiedades, instanciadas por objetos complejos o sistemas, se caracterizan por ser ontológicamente irreductibles (no completamente derivadas) a las propiedades de las partes que constituyen tales objetos y, sin embargo, ontológicamente dependientes de las mismas. Entre casos de entidades con propiedades emergentes, están sociedades respecto de los individuos que las componen, bandadas respecto de los pájaros que las componen, un organismo respecto de las partes biológicas que lo componen, etcétera. Un defensor del nihilismo mereológico debería explicar por qué la existencia de propiedades emergentes fundamentales no sería una buena razón para admitir también la existencia de entidades compuestas. En este sentido, revisar Caves (2015), quien sostiene una interesante defensa de la tesis nihilista en contra de objeciones emergentistas. 
naciones atómicas posibles, cuestión que sobrepoblaría el mundo con millones de piedras allí donde supuestamente encontramos una sola piedra.

En segundo lugar, si un objeto está localizado espacialmente donde sus partes son encontradas a un tiempo dado, entonces resulta ser indeterminado donde ese objeto está exactamente localizado. Un objeto espacial es localizado espacialmente allí donde encontramos su límite. Una mesa puede existir en un lugar $L_{1}$ en $t_{1}$, o bien existir sin una de sus patas en un lugar $L_{2}$ en $t_{2} y$, sin embargo, no podría existir ni en $\mathrm{L}_{1}$ ni en $\mathrm{L}_{2}$ sin tener un límite a lo largo del tiempo que existe. Hay una relación de mutua dependencia ontológica entre un objeto y su límite ${ }^{10}$. Mientas que un límite no puede existir sin ser límite de algo distinto de sí mismo, pues, como sostiene Chisholm, «ellos [los límites] no son entidades por derecho propio; ellos son parasitarios de otras cosas» (1976: 51), un objeto material no puede ocupar un lugar del espacio ni tener una duración en el tiempo sin poseer un límite o, por lo menos, una zona fronteriza que lo separa de sus alrededores espaciales. En su estudio sobre continua y sus límites, Franz Brentano establece lo siguiente:

Cada límite es una conditio sine qua non del continuum en su totalidad. El límite contribuye a la existencia del continuum (1981: 56) [...]. Ningún continuum puede ser concebido aparte de los límites que le pertenecen, como ningún límite puede ser concebido aparte de un continuum, de hecho, aparte de incontables continua a los cuales pertenece como límite. (1914: 201)

Un objeto material no puede existir en el espacio (y el tiempo) sin tener un límite que determine la porción de espacio que ocupa (o el periodo de tiempo que dura). Simultáneamente, un límite es una entidad que no puede existir por derecho propio en el espacio (y el tiempo) y, para lograrlo, requiere de un objeto espacial (y temporal) al cual pertenecer. Por lo tanto, donde quiera que encontremos un objeto en el espacio a un tiempo dado, allí encontraremos también su límite, de modo que «la exactitud de la localización de un objeto está determinada por la localización del límite del objeto» (Casati y Varzi, 1999: 119). Si a lo largo de la superficie de una piedra - en la zona que separa la región espacial ocupada por la piedra y el ambiente espacial donde esta se encuentra- existen, en un momento dado, millones de átomos limítrofes, entonces la localización espacial exacta de la mesa se modificará sutilmente dependiendo de los átomos que consideremos como parte del límite de la región espacial ocupada por la piedra en ese momento.

Si la vaguedad no es ontológica como resultado de la tesis nihilista, entonces es preferible desestimar la existencia de objetos compuestos, puesto que multiplican innecesariamente el mundo con objetos y la localización espacial de objetos materiales resulta indeterminada. Sin embargo, esto no sucede en un mundo en el cual los ítems fundamentales son simples. Si estos son objetos sin partes que nunca componen nada, entonces no puede haber partes limí-

10. En otro artículo (Núñez, 2019a) trabajo con más profundidad la tesis de dependencia ontológica de los límites en la filosofía de Franz Brentano y Roderick Chisholm. 
trofes en sus alrededores espaciales. No hay, por lo tanto, vaguedad ni de la composición ni en la localización. Si x es un objeto simple que está trivialmente compuesto de sí mismo, entonces (i) no hay otros simples limítrofes en la zona donde se ubica x y (ii) la localización espacial de $\mathrm{x}$ es exactamente encontrada en virtud de que x no tiene partes limítrofes. Con los objetos simples, no existe una zona de transición entre el lugar que ocupan en el espacio y sus alrededores, ya que poseen límites precisos (y tampoco pueden tener límites internos dado el rechazo a la DAUP). Si la vaguedad proviene de estructuras mereológicas, los simples no pueden ser vagos. Estos existen completamente en un lugar en un momento particular y sabemos exactamente dónde se encuentra el lugar.

\section{Indeterminación y probabilidad cuántica}

La remoción de la composición material no libera al mundo de la vaguedad. Aunque el nihilismo puede sortear la vaguedad composicional respecto de las circunstancias en las que dos o más objetos componen otro objeto adicional, no le resulta satisfactorio respecto de la localización de un objeto simple. Sin embargo, la idea de que un simple tiene una localización indeterminada $-\mathrm{y}$, por lo tanto, límites vagos - no contradice la tesis nihilista de la composición. Simplemente niega que la vaguedad no tenga cabida en un mundo donde solo existen simples. Esto implica que quien quiera adoptar una posición nihilista respecto de la composición material porque le permite descartar compromisos con una vaguedad ontológica tendrá que considerar que, si bien podrá deshacerse de la vaguedad en la composición, no podrá hacerlo con relación a los límites modales en la localización de los objetos simples. En este aspecto, van Inwagen (1990) sostiene que no hay necesidad de una definición filosófica de la simplicidad. En lugar de propósitos ontológicos o metafísicos, el uso del término simple es meramente en virtud del mundo en el cual sus componentes básicos son definidos por observaciones de la física:

La noción de un simple es una noción funcional, no estructural ni ontológica. El término simple fue introducido en nuestro discurso como un nombre que juega cierto rol en la economía del universo físico. Si hay cosas que juegan este rol, de ningún modo es evidente cuál podría ser su naturaleza. Si la física actual es correcta, entonces parece bastante claro que la categoría simple comprende quarks, leptones y bosones. (van Inwagen, 1990: 158)

Lo que la física contemporánea ha descubierto acerca de las partículas fundamentales desafía el paradigma de la mecánica newtoniana, según la cual cada objeto ocupa un lugar a la vez y se desplaza cubriendo cada punto del espacio a lo largo de su trayectoria. De acuerdo con la física cuántica, las partículas muestran un comportamiento dual onda-partícula que imposibilita conocer con precisión la posición espacial de una partícula. Esta dualidad fue confirmada por el experimento de la doble rendija llevado a cabo por Clinton Davisson y Lester Germer en 1927. Básicamente, este consiste en un lanzador 
de partículas que envía un haz de electrones hacia una placa con dos ranuras verticales y paralelas. Del otro lado, una pantalla indica la posición precisa donde cada electrón se estrella al terminar su viaje después de atravesar las ranuras. Si los electrones se comportan como bolas desplazándose en línea recta, entonces el patrón sobre la pantalla debería replicar el patrón de las ranuras de la placa. Sin embargo, las observaciones evidencian algo completamente diferente. El patrón encontrado en la pantalla muestra que electrones se estrellan con la pantalla en más de dos patrones, entonces, ¿cómo dar cuenta de esta observación? La única explicación es que los electrones no viajan como lo hacen normalmente objetos macroscópicos, sino como ondas sobre el espacio que se sobreponen entre sí formando así un patrón de interferencia sobre la pantalla.

En el desarrollo de la teoría de la dualidad onda-corpúsculo, algunos físicos han postulado diferentes tesis sobre la naturaleza del comportamiento de los objetos cuánticos: exclusivamente ondulatoria (wave-only view), exclusivamente corpuscular (particle-only view) o bien simultáneamente ondulatoria y corpuscular (both-particle-and-wave view). Sin embargo, actualmente, la posición más considerada es que la dualidad onda-corpúsculo hay que entenderla de manera analógica, de modo que la naturaleza del comportamiento de las partículas subatómicas no es ni ondulatorio ni corpuscular (neither-wave-norparticle view), sino que puede exhibir, en diferentes circunstancias de medición y observación, o bien comportamientos de partículas o bien comportamientos de ondas. Incluso desde la teoría de campos cuánticos, que combina elementos de la mecánica cuántica con la teoría clásica de campos físicos electromagnéticos, se plantea que las partículas subatómicas no podrían ser pensadas como entidades propiamente tales. Las realidades físicas fundamentales son en realidad campos cuánticos de los cuales derivan las partículas como fluctuaciones o perturbaciones electromagnéticas ${ }^{11}$.

A diferencia de nuestra experiencia cotidiana con los objetos materiales, el comportamiento espacial de los objetos cuánticos no puede ser descrito como

11. Descubrimientos como este han llevado a filósofos a pensar en toda entidad material como objetos tetradimensionales. Esto es, además de existir en las tres dimensiones espaciales y, por lo tanto, poseer altura, anchura y profundidad, todo objeto material es también una entidad extendida en la dimensión temporal. Así, una mesa es un objeto que ocupa una región del espacio-tiempo que no solamente posee partes físicas (una pata o trozo de cubierta), sino también partes temporales. Esto implica que ningún objeto material existe en su totalidad a un determinado tiempo presente, sino que solo se trata de una parte temporal; la totalidad del objeto debe comprenderse como una suma mereológica de todas sus partes espaciotemporales. Por ejemplo, Mark Heller (2008) considera que los verdaderos componentes ontológicos del mundo son lo que él llama "pedazos de materia tetradimensional» (four-dimensional hunks of matter), los cuales no dependen de ningún tipo de preferencia, decisión o capacidad cognitiva y perceptiva humana. Con este tipo de ontologías perdurantistas, podemos considerar una entidad física, una mesa por ejemplo, literalmente como un evento o proceso espaciotemporal en lugar de un objeto ocupando un lugar en el espacio en momentos diferentes. Espacialidad y duración, por consiguiente, son dimensiones ontológicamente no solo compatibles, sino inseparables a la hora de cuantificar la existencia material. 
una cosa ocupando un solo lugar en diferentes tiempos. Si las partículas subatómicas son fluctuaciones en campos cuánticos, entonces Cox y Forshaw (2011) nos plantean el siguiente desafío: «Construye una teoría de partículas tipo punto de modo tal que esas partículas están también extendidas. Esto no es tan imposible como parece: podemos hacerlo si permitimos a cualquier partícula singular estar en muchos lugares a la vez» (Cox y Forshaw, 2011: 28). De esta manera, en cuanto que perturbaciones de ondas, las partículas no se desplazan ocupando diferentes puntos continuos del espacio en momentos diferentes, sino cubriéndolos todos al mismo tiempo. O bien, más precisamente, una partícula se encuentra simultáneamente en todos aquellos lugares del espacio donde son medibles las diferentes fluctuaciones de onda que puedan ocurrir.

Los físicos denominan función de onda a la representación matemática de la probabilidad de encontrar una partícula en el espacio. A diferencia de objetos-partícula que se encuentran en algún lugar en un instante determinado, objetos-onda están desparramados sobre el espacio mostrando diferentes perturbaciones. La función de onda no nos informa del lugar exacto donde electrones son encontrados en el espacio en un tiempo dado, sino de la probabilidad de su localización: la posibilidad de encontrar un electrón es más alta donde la amplitud de onda es mayor, mientras que es más baja donde la amplitud es menor. Un electrón está extendido a lo largo de una onda y no solo en un lugar por vez, por lo que «cuando decimos que el electrón está en algún lugar dentro de la onda realmente queremos decir que está simultáneamente en todos los lugares en la onda» (Cox y Forshaw, 2011: 31-32). El desplazamiento de un electrón no sigue movimientos rectilíneos de un lugar a otro, sino que barre el espacio a través de todas las trayectorias posibles simultáneamente.

Una consecuencia de la dualidad onda-partícula es el denominado principio de incertidumbre. Este establece la imposibilidad de conocer al mismo tiempo la posición y el momentum de una partícula: como más información tenemos sobre la localización de una partícula en algún lugar en un tiempo inicial, menos información tenemos para medir y predecir la velocidad con la que se desplaza. El mejor intento que pueden hacer los físicos es predecir la probabilidad que tiene una partícula cuántica de estar en un lugar, por lo que resulta indeterminada su ocupación precisa en el espacio. Sin embargo, mientras que las probabilidades de la localización son limitadas, alguna información acerca de la velocidad es inevitablemente perdida ( $\mathrm{y}$ viceversa). Esta incertidumbre no es debida ni al conocimiento ni a la imprecisión del lenguaje matemático, sino a la naturaleza misma. En palabras de Cox y Forshaw:

No es el caso que podemos solamente predecir la probabilidad de una partícula de estar en un lugar u otro porque seamos ignorantes. No podemos, ni siquiera en principio, predecir la posición que una partícula tendrá. Lo que podemos predecir, con absoluta precisión, es la probabilidad de que una partícula sea encontrada en un lugar particular si lo buscamos. El progreso teórico y expe- 
rimental indica que la naturaleza realmente utiliza números aleatorios, y la pérdida de certeza en predecir las posiciones de las partículas es una propiedad intrínseca del mundo físico. (2011: 45)

\section{Límites y la vaguedad modal de la simplicidad}

¿Qué consecuencias teóricas podrían traer los descubrimientos de la física cuántica acerca de la simplicidad metafísica? En primer lugar, la idea de objetos simples como entidades que ocupan una porción limitada de espacio en un tiempo dado es desafiada por una ontología de partículas subatómicas. A diferencia de un objeto físico ordinario, una fluctuación de onda es un tipo de entidad o evento material en el cual la idea de límite es difusa o ambigua. Un electrón, dado su comportamiento ondulatorio, está extendido en el espacio sin localizarse solamente en un lugar a la vez. En este sentido, la noción de límites está dada tanto por la amplitud máxima (es decir, punto alcanzado en la cresta de una onda) como por la longitud de onda (es decir, la distancia entre las crestas de dos ondas continuas). En este caso, no existiría vaguedad en la medida en que podemos hacer cálculos matemáticos precisos para los valores de ambos límites. Sin embargo, aunque pueda tener sentido esta representación sobre cómo entender los límites de un objeto onda-partícula, resulta aún muy distante y ajena a nuestra comprensión ordinaria de los límites de objetos materiales.

Los simples no muestran vaguedad ni de composición ni de localización debido a sus límites precisos por la carencia de una estructura mereológica. Sin embargo, esta imagen de la simplicidad es diluida en la medida en que partículas cuánticas tienen un rol fundamental en su comprensión. Junto con Heller (2008), considero que los objetos materiales poseen límites espaciales, temporales y modales: los lugares donde están localizados, los tiempos que duran y los mundos donde existen. Hablar de límites modales no es tan claro como hablar de límites espaciales o temporales. No obstante, entiendo por la expresión límites modales el hecho de que un objeto puede estar extendido a lo largo de mundos posibles tanto como en el espacio y el tiempo. La expresión se refiere a si acaso un objeto posee algunas de sus propiedades esencialmente, es decir, en cada uno de los mundos posibles donde existe:

Preguntar acerca del límite modal de una cosa es realmente preguntar acerca de sus propiedades esenciales. Esto es análogo al hecho de que preguntar acerca de los límites temporales es, en efecto, preguntar acerca de sus condiciones de persistencia. (Heller, 2008: 72)

La vaguedad aplica a los límites espaciales, temporales y modales de los objetos ordinarios físicos. ¿Tiene un objeto material su localización espacial, partes, extensión temporal y propiedades en cada mundo en el que existe? ¿Cuál es la configuración atómica esencial que un objeto debe tener para persistir temporalmente? ¿Cuáles son las propiedades esenciales que permiten a un obje- 
to existir en todos los mundos posibles en los que existe? En física cuántica, no hay ni un lugar exacto donde una partícula es localizada ni un tiempo preciso cuando encontrarla y, por consiguiente, lo mejor que podemos hacer es predecir sus probabilidades. En este aspecto, el tipo de vaguedad de la simplicidad no surge de un límite a la composición, sino de la indeterminación de sus límites modales, es decir, el límite trazado para determinar cuál es el mundo actual entre todos los mundos posibles en los que un objeto existe.

Puesto que una partícula cuántica tiene una probabilidad similar de ser encontrada aquí o allá a lo largo de una onda (es decir, se mueve a través de todas las trayectorias posibles simultáneamente), podemos pensar esta probabilidad en términos de los mundos posibles donde una partícula puede ser localizada en un instante. No obstante, ¿cómo podemos trazar un límite modal preciso para determinar qué mundo debería ser $e l$ mundo donde una partícula está ahora localizada? Si la naturaleza misma no es precisa respecto de dónde un simple puede ser encontrado en un instante dado y, más bien, solo nos entrega posibilidades de localización, entonces no hay un límite modal preciso para determinar si este mundo o ese mundo instancia su posición actual.

Imagina una pequeña bola de goma (llamada Ball). De acuerdo con los postulados de la física cuántica, Ball es un simple que rebota velozmente de un lugar a otro dentro de una caja. Según el principio de incertidumbre, si recopilamos más información sobre el lugar donde Ball está localizada en un instante, menos información obtenemos de lo tan rápido que se mueve de ese lugar a otro. Puesto que Ball se comporta como una onda, no se encuentra simplemente localizada en un lugar a la vez, sino en todos los lugares de la caja. De este modo, tenemos un rango de probabilidades de todas las trayectorias posibles de Ball. En términos modales, existen muchos mundos posibles (con probabilidades similares) donde Ball se encuentra en el interior de la caja a un tiempo dado. La localización de Ball es, por consiguiente, indeterminada en la medida en que la naturaleza no nos entrega información precisa para preferir objetivamente qué mundo es el mundo actual de su posición. No podemos escoger solamente un mundo, ya que Ball se encuentra extendida a lo largo de todos ellos. Lo que sabemos con certeza es que la trayectoria de Ball no puede ser medida al tomar todos los puntos que Ball ocupa en momentos diferentes. En rigor, su trayectoria es la suma de los mundos donde Ball tiene mayores posibilidades de ser encontrada. No podemos indicar un solo punto de la localización de Ball al interior de la caja porque Ball está literalmente en todas partes a la vez. Esto, sin embargo, es bastante vago. Si tus llaves están perdidas en tu casa y preguntas "¿alguien sabe dónde están mis llaves?», y otra persona responde "sí, lo sé, están probablemente en todos los rincones de la casa», entonces tendrías derecho a sentirte decepcionado por lo impreciso de la respuesta.

La vaguedad de la simplicidad no puede ser encontrada en la composición porque un simple está trivialmente solo compuesto de sí mismo en todos los mundos donde existe. Por consiguiente, un simple es un objeto vago debido 
a la pluralidad de las posibles regiones espaciales que puede ocupar: las probabilidades para un simple de ocupar esta región espacial y esa región espacial son similares. La vaguedad, por lo tanto, surge de los límites modales de la simplicidad. Si pensamos un simple como las partículas fundamentales de la física, entonces podemos pensarlo como un objeto modalmente extendido a través de mundos posibles en lo que respecta a sus probabilidades de localización. Si resulta ser indeterminado si una región espacial es ocupada por materia, entonces no podemos trazar un límite modal que no sea arbitrario para establecer claramente el mundo en el cual una región espacial determinada es, de hecho, ocupada por un objeto simple.

Este tipo de vaguedad de la simplicidad es explicada también a partir de la definición de un objeto simple dada por Peter Simons: «Una entidad sin partes genuinas cuyo locus tiene partes genuinas» (2014: 63). Esta tesis de la simplicidad está basada en rechazar lo que el propio autor denomina the geometric correspondence principle (GCP), según el cual cualquier objeto extenso tiene partes genuinas que se corresponden con las partes o subregiones de la región espacial que ocupa. Hay una equivalencia geométrica entre la estructura mereológica del espacio y la estructura mereológica de los objetos materiales. Una definición del GCP es la siguiente:

Un objeto material $x$ ocupa geométricamente la región espacial $R$ en un tiempo $t=d f$ cada parte de x ocupa exactamente cada para de $\mathrm{R}$ en $\mathrm{t}$.

Un objeto simple parece seguir fácilmente el GCP: puesto que es un átomo mereológico (es decir, no muestra una estructura todo-partes), la región de espacio que le corresponde geométricamente ocupar no tiene subregiones. Según Simons, el locus es la región de espacio que un objeto extenso ocupa. Sin embargo, para una partícula cuántica, las probabilidades de encontrar su locus están distribuidas en cada subregión de espacio donde se desplaza. Así, un objeto simple o partícula cuántica p no tiene partes físicas que se correspondan en un tiempo $t$ en la región espacial R; más bien $\mathrm{p}$ es un átomo mereológico que ocupa modalmente $\mathrm{R}$ en $\mathrm{t}$. Entonces, podemos plantear la siguiente definición:

Un objeto simple ocupa $a_{\text {modalmente }}$ la región espacial $R$ en un tiempo $t={ }_{d f}$ existe un mundo posible para cada subregión de R que es completamente ocupada por $\mathrm{x}$ en $\mathrm{t}$.

Según lo anterior, $\mathrm{x}$ no ocupa cada una de las subregiones de $\mathrm{R}$ en tiempos diferentes; más bien $\mathrm{x}$ existe extendido sobre $\mathrm{R}$ al ocupar cada una de sus subregiones simultáneamente en la medida en que todas ellas existen modalmente en un mismo tiempo. Así, dado un objeto simple $\mathrm{x}$, las subregiones Rs de una región espacial $\mathrm{R}$, los mundos posibles Ms y un tiempo t, entonces:

$\mathrm{x}$ ocupa $\mathrm{a}_{\text {modalmente }} \mathrm{R}$ en t si y solo si (i) $\mathrm{x}$ se extiende espacialmente sobre $\mathrm{R}$ al ocupar cada uno de los Rs en t, (ii) cada uno de los Rs es completamente 
ocupado por x en t, y (iii) cada uno de los Rs que x ocupa en t corresponden a cada uno de los Ms.

Entonces, podemos definir un simple como un objeto sin estructura mereológica modalmente extendido sobre las diferentes subregiones de la región espacial que ocupa. La violación del GCP en la simplicidad nos muestra que un objeto simple no tiene una relación geométrica con el espacio, sino una relación modal con el mismo. Esto último deja la puerta abierta para la vaguedad ontológica en la simplicidad en la medida en que no existe un límite preciso para escoger un mundo posible en desmedro de otros mundos que precisen la localización espacial de un simple.

Este tipo de vaguedad modal de la simplicidad es también considerada por Katherine Hawley (2004). Ella acepta que, si resulta ser indeterminado que en algunas ocasiones un punto este ocupado con materia, entonces la simplicidad admite la vaguedad modal. Por lo tanto, en el caso de la TG antes revisada, la vaguedad surge para cada punto espacial en la medida en que es indeterminado si es exactamente ocupado por un objeto-punto material. Por otro lado, si consideramos MaxCon como la tesis correcta de la simplicidad, entonces la vaguedad modal surge «si un objeto posible dado es el objeto actual, o, a partir de la vaguedad en cuanto a si el objeto posible relevante es dividido" (Hawley, 2004: 399). Si es indeterminado que un punto en el espacio se encuentre ocupado (saturado de materia), entonces sería vago si un objeto está dividido, es decir, si es continuo o discontinuo:

Imagina a un objeto en forma de un sándwich de queso en el cual las rebanadas de pan corresponden a regiones determinadamente rellenas de materia, y el queso corresponde a una región indeterminadamente rellena de materia. La región en forma de sándwich es continua, pero la región en forma de pan es discontinua. Ahora, imagina un objeto que exactamente ocupa la región en forma de sándwich si esa región puede ser llenada de materia, y exactamente ocupa la suma discontinua de las dos regiones en forma de rebanadas de pan. Si es vago si la región en forma de queso contiene materia, entonces es vago si el objeto en cuestión es dividido. (Hawley, 2004: 399)

Si un objeto ocupa una región de espacio al llenar cada punto espacial que compone dicha región y es indeterminado, dadas las características modales de los objetos simples, si algunos de esos puntos contienen actualmente materia, entonces es vago si un objeto ocupa continuamente el espacio o bien presenta interrupciones que lo podrían hacer divisible. De acuerdo con MaxCon, un objeto simple tiene una determinada y homogénea distribución de la materia sin baches o regiones de espacio sin ocupar: un objeto maximalistamente continuo que ocupa exactamente una región de espacio que no es parte de una región de espacio más extensa. Hawley argumenta que si, por una parte, $\mathrm{x}$ es la región de espacio más extensa que determinadamente contiene materia de un objeto $\mathrm{O}$, mientras que, por otro lado, y es la región de espacio más extensa que indeterminadamente contiene mate- 
ria de $\mathrm{O}$, entonces surge una vaguedad de la identidad. Es indeterminado que un objeto llene $\mathrm{x}$ con materia, pero indeterminado si un objeto llene y con materia. Si es así, resulta indeterminado tanto si $\mathrm{O}$ es idéntico a x (es decir, a un objeto simple que ocupa maximalistamente una región continua de espacio) como si $\mathrm{O}$ es idéntico a la suma o fusión mereológica entre x e y (es decir, un objeto compuesto). Así, si consideramos la incertidumbre de la localización como una característica de la dualidad onda-partícula en la física cuántica, las respuestas a la pregunta por la simplicidad aún poseen componentes de vaguedad.

Aunque la tesis nihilista elude el problema de la vaguedad composicional en cuanto que no existen objetos limítrofes, un mundo constituido únicamente por objetos simples no erradica la vaguedad ontológica. Esta no proviene de límites espaciales ni temporales, sino de la vaguedad del límite modal respecto del mundo que actualiza la localización exacta de un objeto simple. Tal como Hawley sostiene, «respuestas a la pregunta por la simplicidad son supuestamente verdades metafísicas necesarias, verdadero en mundos improbables tal modo como consideramos ser el mundo actual» (2004: 402). Sin embargo, el universo en cuanto tal es un lugar vago en el cual lo mejor que podemos hacer es predecir los mundos posibles donde los objetos simples de la materia pueden ser encontrados con más probabilidad. Siguiendo la tesis nihilista, los límites espaciales y temporales de los objetos simples pueden ser muy precisos, sin embargo, modalmente muy vagos e indeterminados.

\section{Referencias bibliográficas}

Brentano, Franz (1914). Philosophical Investigations on Space, Time and the Continuum (trad. Barry Smith). Nueva York: Routledge, 1988.

Casati, Roberto y Varzi, Achilles (1999). Parts and Places: The Structure of Spatial Representation. Cambridge (Massachusetts): MIT Press.

Caves, Richard (2015). «Emergence for Nihilists». Pacific Philosophical Quaterly, 99 (1), 2-28.

Chisholm, Roderick (1976). Person and Object: A Metaphysical Study. Illinois: Open Court Publishing.

Cowles, David y White, Michael (1991). "Vague Objects for Those Who Want Them». Philosophical Studies, 63 (2), 203-216.

Cox, Brian y Forshaw, Jeff (2011). The Quantum Universe: Everything that Can Happen does Happen. Nueva York: Allen Lane.

Dummett, Michael (1999). «Wang's Paradox». En: Keefe, Rosanna y Smith, Peter (eds.). Vagueness: A Reader, 99-118. Massachusetts: MIT Press.

Evans, Gareth (1978). «Can There Be Vague Objects?». Analysis, 38, 208.

Hawley, Katherine (2004). "Borderline Simple or Extremely Simple». The Monist, 87 (3), 385-404. <https://doi.org/10.5840/monist200487319>

Heller, Mark (2008). The Ontology of Physical Objects: Four-Dimensional Hunks of Matter. Cambridge: Cambridge University Press. 
Inwagen, Peter van (1990). Material Beings. Londres: Cornell University Press.

- (2001). «The Doctrine of Arbitrary Undetached Parts». En: van Inwagen, Peter (ed.). Ontology, Identity, and Modality. Cambridge: Cambridge University Press, 75-94.

Lewis, David (1986). On the Plurality of Worlds. Oxford: Blackwell.

Lowe, E. J. (1999). «Vague Identity and Quantum Indeterminacy: Further Reflections». Analysis, 59, 328-330.

Markosian, Ned (1998). «Simples». Australasian Journal of Philosophy, 76 (2), 213-228.

<https://doi.org/10.1080/00048409812348361>

McDaniel, Kris (2007). «Brutal Simples». En: Zimmerman, Dean (ed.). Oxford Studies in Metaphysics, III. Oxford: Oxford University Press, 233265.

Merricks, Trenton (2001). Objects and Persons. Oxford: Oxford University Press.

<https://doi.org/10.1093/0199245363.001.0001>

NuñEz, Gonzalo (2019a). «Boundaries and things: a metaphysical study of the Brentano-Chisholm theory». Kriterion - journal of philosophy, 33 (2), $1-34$.

- (2019b). «La pregunta por la composición material como una pregunta sobre límites». Estudios de Filosofía, 59, 97-120. <https://doi.org/10.17533/udea.ef.n59a05>

Russell, Bertrand (1918). The Philosophy of Logical Atomism. Illinois: The Philosophy of Logical Atomism, 1994.

- (1905). «On Denoting». Mind, 14, 479-493.

- (1923). «Vagueness». En: Keefe, Rosanna y Smith, Peter (eds.). Vagueness: A Reader. Massachusetts: MIT Press, 1999, 61-68.

Simons, Peter (1991). «Faces, Boundaries, and Thin Layers». En: MarTinich, A. P. y White, Michael (eds.). Certainty and Surface in Epistemology and Philosophical Method: Essays in Honor of Avrum Stroll (Problems in Contemporary Philosophy). Wales: The Edwin Mellen Press.

- (2014). «Where It's At: Modes of Ocupation and Kinds of Ocupant». En: S. Kleinschmidt (ed.). Mereology \& Location. Oxford: Oxford University Press, 59-68.

Unger, Peter (1980). "The Problem of Many». Midwest Studies in Philosophy, 5, 411-467. <https://doi.org/10.1111/j.1475-4975.1980.tb00416.x>

Wittgenstein, Ludwig (1921). Tractatus Logico-Philosophicus. Madrid: Alianza, 2004. 
Gonzalo Núñez Erices es licenciado (2008) y magíster (2010) en filosofía por la Universidad Alberto Hurtado (Chile), y doctor en filosofía (2018) por la University of Sheffield (Reino Unido), este último título financiado por Becas Chile, otorgadas por el gobierno de Chile en una postulación pública. Actualmente, es docente e investigador del Departamento de Filosofía de la Universidad Católica del Maule, Chile. Su interés de investigación se centra en metafísica en la tradición analítica y el pensamiento de Ludwig Wittgenstein. Sus publicaciones previas son, en gran medida, sobre límites, ontología de objetos materiales y filosofía del lenguaje, entre otras temáticas.

Gonzalo Núñez Erices has a BA (2008) and Master's (2010) in Philosophy from the Universidad Alberto Hurtado (Chile), and a PhD in Philosophy (2018) from the University of Sheffield, with a Becas Chile grant awarded by the government of Chile. Currently, he is an academic and researcher at the Philosophy department of the Universidad Católica del Maule, Chile. His research interests focus on the analytic tradition of metaphysics and the philosophical work of Ludwig Wittgenstein. His previous publications are mostly about boundaries, the ontology of material objects, and the philosophy of language, among other topics. 